\title{
LA TRANSFORMACIÓN DE LAS TECNOLOGÍAS DE LA INFORMACIÓN Y LA COMUNICACIÓN EN TECNOLOGÍAS DE LA EDUCACIÓN : componentes de un camino incierto
}

\author{
Transformação das tecnologias da informação \\ e comunicação em tecnologias da educação: \\ componentes de uma trajetória incerta
}

\author{
Juana María Sancho
}

Professora Doutora em Tecnologia Educativa e Coordenadora do grupo de investigação consolidado FINT, Catedrática da Universidad de Barcelona, Espanha, e-mail: jmsancho@ub.edu

\section{Resumen}

En los últimos años, el uso de las computadoras y los distintos desarrollos vinculados a las tecnologías digitales de la información y la comunicación han sido presentados no sólo como las herramientas por excelencia para la mejora de la educación, sino como factor de modernización de los sistemas educativos. Sin embargo, el seguimiento delos distintos proyectos llevados a cabo y de las inversiones realizadas, a menudo a costa de deuda externa, no han podido evidenciar que su uso en la escuela signifique una mejora inmediata de los procesos de enseñanza y

Rev. Diálogo Educ., Curitiba, v. 9, n. 28, p. 651-669, set./ dez. 2009 
aprendizaje y de los resultados de los estudiantes. A partir de esta constatación. La primera parte de esta ponencia analizo y problematizo el discurso que inviste a estas tecnologías de poderes especiales para la mejora de la educación, a la vez que configuro los cambios que la ubicuidad de estas tecnologías en la vida cotidiana de niños, niñas y adolescentes están representando en su forma de aprender, de enfrentarse a la información y de valorar el conocimiento. En la segunda, a partir de evidencias aportadas por la investigación, dimensiono los factores asociados al cambio que los sistemas educativos y las escuelas tendrían que promover para convertir las TIC en Tecnologías de la Educación.

Palabras-clave: Tecnología educativa. Cambio educativo. Innovación. Política educativa. Formación del profesorado.

\section{Resumo}

Nos últimos anos, o uso de computadores e os diversos desenvolvimentos relacionados com as tecnologias digitais de informação e comunicação têm sido apresentados, não apenas como instrumentos por excelência para a melhoria da educação, mas como um elemento determinante na modernização de sistemas educação. No entanto, o acompanhamento de vários projetos realizados e os investimentos, muitas vezes à custa da dívida externa, não foram capazes de demonstrar que a sua utilização nas escolas irá significar uma imediata melhoria do ensino e da aprendizagem, os processos e os resultados dos alunos. A partir dessa contatação é analisado e problematizado o discurso que investe as tecnologias de poderes especiais para melhorar a educação, ao passo que também configura as mudanças que a onipresença destas tecnologias na vida diária de crianças e adolescentes estão representando em sua forma de aprender, de lidar com a informação e de valorizar o conhecimento. Em seguida, a partir das evidências produzidas pela investigação, são dimensionados os fatores associados à mudança que os sistemas de ensino e as escolas teriam que promover para converter as TIC em tecnologias da educação.

Palavras-chave: Tecnologiaeducacional. Mudançaeducativa. Inovação. Política educacional. Formação de professores.

Rev. Diálogo Educ., Curitiba, v. 9, n. 28, p. 651-669, set./ dez. 2009 
La transformación de las tecnologías de la información y la comunicación en tecnologías de la educación

\section{Desde dónde hablo}

En 1982, cuando las computadoras eran poco más que potentes máquinas de calcular, fui invitada a participar en el primer programa de informática educativa llevado a cabo en E spaña, a iniciativa de un grupo de profesores pioneros del ámbito de la Formación Profesional. ${ }^{1} \mathrm{D}$ esde el principio me interesó analizar por qué estas máquinas estaban levantando tantas expectativas y comencé a estudiar tanto los discursos sobre su potencial, como su incidencia en la práctica. Y desde el primer momento empecé a vislumbrar una gran distancia entre lo que se anunciaba como posible y lo que llegaba a ser real.

Como había sucedido con otros desarrollos tecnológicos (radio, cine, televisión, vídeo,... ) cadanuevo avanceen las tecnologías (electrónicas o digitales) de la información y la comunicación se anunciaba (y se sigue anunciando) como la panacea, como el recurso clave para solucionar los problemas de la educación (SANCHO, 1998) y en cada nuevo discurso inflamado (en el fondo siempre repetido) sobre el poder educativo de éstas, yo encontraba nuevas claves para repensar la educación. Así he mantenido mi interés por un campo de estudio que me permite seguir la evolución y los fenómenos creados por una tecnología en permanente expansión, que está contribuyendo a transformar todas las formas devida. Pero que, sobre todo, melleva estudiar la dificultad de los sistemas educativos introducir cambios que les permitan responder mejor a los desafíos actuales de la educación.

\section{INTRODUCCIÓN}

En los últimos años, el desarrollo exponencial de las tecnologías digitales delainformación y la comunicación; su ubicuidad en prácticamente todos los ámbitos de la sociedad: producción, investigación, trabajo, ocio... ; y la rapidez con la que han pasado constituirse en el entorno natural de niños, niñas y jóvenes, han generado unas altas expectativas sobre su papel en la mejora de los resultados del aprendizaje escolar. D esde la década de1970, el movimiento delos denominados doomsters(BIG UM;KENWAY, 1998²), los entusiastas defensores de quela computadora representala quinta esencia de la innovación educativa e incluso un nuevo paradigma para la

Centro de Recursos de Informática Educativa y Profesional (CRIEP).

2 Estos autores identificaron cuatro posiciones o discursos en relación al uso educativo de las TIC a cuyos seguidores denominaron: boosters-propulsores entusiastas-; doomstersfatalistas-; desescolarizadores y críticos.

Rev. Diálogo Educ., Curitiba, v. 9, n. 28, p. 651-669, set./ dez. 2009 
educación, no han dejado de magnificar el potencial educativo de estas tecnologías. Aunque en este momento, la persistente falta de evidencias sobre el impacto real de estas herramientas en la mejora del aprendizaje escolar ${ }^{3}$ haga difícil mantener las afirmaciones contundentes dereconocidos entusiastas de este movimiento como Seymour Papert, para quien el único fallo de las ideas pedagógicas progresistas de principios de siglo XX había sido no contar con computadoras. "D ewey, Montessori, yNeill propusieron educar a los niños con un espíritu para mí fundamentalmente correcto pero quefalló en la práctica por una falta de base tecnológica. A hora el ordenador la proporciona (PAPERT, 1979, p. 85).

O como las de Perelman (1992, p. 23-27) al dotar a las computadoras de la capacidad de hacer más inteligentes a los humanos y considerar que la interacción entre los individuos y las máquinas está configurando procesos de aprendizaje que pueden llevar a poder (y tener que) prescindir del contexto institucional de la escuela.

Llamo a la nueva ola de tecnología hiperaprendizaje, (HA). Se trata no sólo de un artilugio o proceso, sino de un universo de nuevas tecnologíasqueposeen yaumentan lainteligencia. Hiper de hiperaprendizaje se refiere no sólo a la velocidad extraordinaria y el alcance de las nuevas tecnologías de la información, sino aun grado sin precedentes de conectividad del conocimiento, la experiencia, los medios, y los cerebros tanto humanos como no humanos. El aprendizaje en HA se refiere literalmente a la transformación del conocimiento y el comportamiento através dela experiencia-lo queel aprendizaje significa en este contexto va más alládela mera educación o el adiestramiento del mismo modo que la lanzadera espacial va más allá que la canoa de tronco de árbol. [... ] De forma más significativa, la "enseñanza" y el "aprendizaje" se funden y transforman en hiperaprendizaje: maquinas ayudando a los humanos a aprender. Humanos ayudando a las máquinas a aprender. Nadielo llama piensa en ello como "educación" 0 "adiestramiento". No hay "escuela.

Lo que sí sigue parece seguir vigente es la demoledora crítica realizada por este autor, a partir de su propia experiencia como profesor, a la falta de capacidad y voluntad de los sistemas educativos (integrados por la Administración, los supervisores, los profesores, los asesores, los

3 Ver, entre otros, BALANSKAT; BLAMIRE; KEFALA (2006); JÄRVELÄ (2006); KOZMA (2003).

Rev. Diálogo Educ., Curitiba, v. 9, n. 28, p. 651-669, set./ dez. 2009 
La transformación de las tecnologías de la información y la comunicación en tecnologías de la educación

estudiantes y la propia sociedad) para dar respuestas a las necesidades educativas emergentes en un mundo cada vez más saturado de tecnologías digitales y de información.

Mi lucha durante ese año para dar respuesta a las necesidades de aprendizaje de los jóvenes estudiantes mientras librabaun combate continuo con los mandatarios "matadores-de-mentes" de una burocracia educativa corrupta galvanizó mi desdén por el personal académico del mismo modo que otras experiencias similares lo hicieron a otros escritores y profesores apóstatas tales como John Holt, Jonathan Kosol, and Pat Conroy. (PERELMAN, 1992, p. 7-8).

De hecho, como argumento más adelante, los principales obstáculos para convertir a las tecnologías de la información y la comunicación en tecnologías de la educación se encuentran en el tupido entramado de burocracia y cultura inercial que los sistemas educativos han ido tejiendo a través de los años (TYACK; TOBIN, 1994).

Sin embargo, la fascinación que despiertan estas herramientas no ha decaído. El 5 de septiembre de 2008, apareció en el diario español El País la noticia-anuncio de la comercialización de una computadora para niños, "diseñada para mejorar la capacidad de aprendizaje de los usuarios más jóvenes y de los estudiantes de primaria a través de la diversión y la interacción." En el suplemento de Negocios de ese mismo diario, aparecía el 14 de septiembre un artículo de Jeffrey D. Sachs, catedrático de Economía y director del Instituto de la Tierra de la Universidad de Columbia, en el que defendía la telefonía móvil como arma contra la pobreza y argumentaba que con el uso de los teléfonos celulares "en todo el mundo, los colegios de todos los niveles se volverán glo bales, y se unirán en redes de educación digitales de todo el mundo. Los niños de Estados Unidos no sólo aprenderán sobre África, China e India en libros y vídeos, sino también mediante enlaces directos entre aulas situadas en diferentes partes del mundo."

Por su parte, las ideas descolarizadoras se están abriéndose nuevas vías a través de las propuestas variadas de educación a distancia, esta vez basadas en el glamour de Internet y sus ilimitados recursos. D e hecho, tres de los posibles escenarios identificados por el proyecto de la OCDE sobre L a escuela del mañana, tienen un claro perfil desescolarizador y en los tres se confía en el papel de las tecnologías digitales para dar respuesta a las necesidades educativas de la población.

Rev. Diálogo Educ., Curitiba, v. 9, n. 28, p. 651-669, set./ dez. 2009 
Los tres se basan en las dificultades que encuentra la escuela actual para llevar a cabo su tares y satisfacer las demandas de los estudiantes y las familias. El primero, denominado como escenario del modelo de mercado extendido, está en relación con el descontento de las familias con la escuela y la presión de las corporaciones por encontrar nuevos nichos de negocio; el segundo, denominado de las redes de aprendizaje y la sociedad de la red, recoge las ideas más progresistas y solidarias sobre el desarrollo de Internet y las conecta con la idea de redes sociales de aprendizaje de los años 1970; el tercero, el del éx odo de docentes y desintegración del sistema, representa el imposibilidad de los sistemas escolares para lograr que buenos profesionales se dediquen a la educación (ISTANCE, 2006)

En los últimos años el discurso fatalista basado en los peligros que la utilización de las herramientas digitales puede comportar para niños, niñas y jóvenes se ha atemperado; si bien se han consolidado y ampliado las posiciones críticas:

- alertando de la necesidad de tener en cuenta los componentes del sistema educativo como un todo y de encuadrar estas tecnologías en experiencias de aprendizaje que realmente merezcan la pena, si realmente se pretende transformar y mejorar la educación (SANCHO ; HERNÁND EZ, 2006);

- evidenciando cómo algunos sistemas educativos, en su afán de presentarse como modernos e innovadores, están desviando lainversión en bibliotecasy servicios escolaresfundamentales (programas de salud, de enseñanza de lenguas, de atención a la diversidad, etc.) para comprar unas máquinas que al carecer de las condiciones necesarias para sacarles partido terminan por hacerse obsoletas antes de ser utilizadas (OPPENHEIMER, 2004);

- llamando la atención sobre la necesidad de invertir en formación del profesorado para que el gasto considerable que representa la compra de equipamiento digital no termine en una mayor trivialización de los procesos de enseñanza, al ocupar a los estudiantes en tareas como el scooping ${ }^{4}$ y el smushing ${ }^{5}$ que favorecen poco el desarrollo de las habilidades de pensamiento de orden superior (McKENZIE, 2007).

Recoger datos con rapidez.

5 Smushing es una manera informal de referirse a la fusión de bases de datos de conocimiento meramente identificatorio.

Rev. Diálogo Educ., Curitiba, v. 9, n. 28, p. 651-669, set./ dez. 2009 
La transformación de las tecnologías de la información y la comunicación en tecnologías de la educación

Por otro lado, en estos últimos años, independientemente de lo que sucede en los sistemas educativos, las TIC han impregnado de tal manera los sistemas sociales, las relaciones y los hábitos individuales y sociales, que han llegado a configurar un nuevo mundo que niños, niñas y jóvenes han pasado a habitar, como no podía ser de otro modo, con toda naturalidad. Esta situación ha llevado a autores como John Perry Barlow, ${ }^{6}$ a denominar nativos digitales a quienes han nacido y crecido en la era de Internet, los espacios digitales y las nuevas tecnologías de la información y la comunicación. Estos niños, chicos y chicas chocan con sus profesores y profesoras, padres y madres, tecnológicamente analfabetos, que para este mismo autor, han emigrado a este entorno siendo por ello inmigrantes digitales. Los nativos y los inmigrantes digitales parecen tener una configuración mental y una forma de interactuar con el mundo y con la información bastante diferentes.

De hecho, como he argumentado en un trabajo anterior (SANCHO, en prensa), en la siempre difícil tarea de caracterizar las sucesivas generaciones de individuos Howe y Strauss (2000) han denominado M illennials ${ }^{7}$ a los nacidos desde 1980 y que han crecido en un contexto en el que las tecnologías digitales son parte consustancial de su vida diaria. Estos individuos pertenecen a las primeras generaciones que se han desarrollado en un entorno mediado por recursos digitales en el que la mayor parte de sus actividades relacionadas con la comunicación entre pares y la gestión de conocimiento -en sentido amplio- están mediadas por estas tecnologías. Se cree que los M illennials tienen una gran habilidad para manejar las computadoras, son creativos con las tecnologías y, sobre todo, tienen un gran talento para realizar varias tareas a la vez en un mundo en el que la ubicuidad de las conexiones tecnológicas se da por sentado. O tros autores también se han referido a estas generaciones como G eneración IM - generación del mensaje instantáneo (LENHART; RAINIE ; LEWIS, 2001); generación homo zappiens (VEEN; VRAKKING, 2006), por su habilidad para controlar al mismo tiempo diferentes fuentes de información digital; generación de la red (OBLINGER; OBLINGER, 2005), o la generación jugadora - $\mathrm{G}$ amer $\mathrm{G}$ eneration- (CARSTENS; BECK, 2005), por su pasión por los videojuegos. En general, los individuos pertenecientes a estas

Accesible at: <http:/ / en.wikipedia.org/ wiki/John_Perry_Barlow>.

7 In 2006 el Centro de Investigación e Innovación Educativa (CERI) de la OCDE, comenzó un proyecto de investigación y desarrollo denominado Los aprendices del nuevo milenio. Accesible at: <http:/ / www.oecd.org/ document/ 10/ 0,3343,en_2649_35845581_38358154_1_1_1_1,00.html>.

Rev. Diálogo Educ., Curitiba, v. 9, n. 28, p. 651-669, set./ dez. 2009 
generaciones consideran que la multitarea es la aproximación normal al uso de los medios digitales: estar conectados a Internet, mientras miran la TV, hablan por teléfono y hacen los deberes. Su continuada actividad con estas tecnologías ha configurado de manera fundamental su noción de comunicación, gestión del conocimiento, su forma de aprender, así como sus valores personales y sociales.

Incluso considerando la importante brecha digital que divide a los inforricos de los infopobres, convirtiéndose en un elemento más de la discriminación y falta de equidad social que asola el mundo actual, una parte importante de los niños, niñas y jóvenes actuales - excepto los sometidos a niveles de gran pobreza que han de emplear toda su energía al arte de sobrevivir en condiciones extremas- pertenecen a las generaciones anteriormente descritas. Sin embargo los sistemas educativos tienden a desconocer o desconsiderar no sólo sus saberes y habilidades sino también sus intereses y sus formas de aprender.

El entorno tecnológico en el que se mueven con gran comodidad los niños, niñas y jóvenes actuales ha dado nuevos argumentos a los entusiastas de las tecnologías digitales para propiciar su integración en la escuela. Sin embargo, convertir estas tecnologías en tecnologías de la educación requiere mucho más que inversiones parciales, por millonarias que sean.

\section{DESARROLLO}

Como he apuntado en la introducción, en estos momentos parece existir un acuerdo generalizado, a pesar de las muchas cautelas, sobre la necesidad, conveniencia o imponderabilidad de considerar el uso de las TIC en los procesos de enseñanza y aprendizaje. Para autores como Järvelä(2006), los mejores argumentos, basados en lainvestigación y en la experiencia práctica, para la implementación de las TIC en la educación se apoyan en los siguientes principios.

- las TIC pueden aumentar la autenticidad y el interés;

- las TIC pueden construir comunidades entre diferentes escuelas, grupos colaborativos y profesores;

- las TIC pueden ayudar a compartir perspectivas entre estudiantes con diferentes conocimientos; proporcionando apoyo entre pares y dando apoyo a "experiencias pioneras" en distintos campos;

Rev. Diálogo Educ., Curitiba, v. 9, n. 28, p. 651-669, set./ dez. 2009 
La transformación de las tecnologías de la información y la comunicación en tecnologías de la educación

- lasTIC facilitan el uso los modelos orientados alaindagación y la resolución de problemas con apoyo tecnológico para aumentar la capacidad de aprender a aprender;

- las TIC proporcionan formas innovadoras (por ejemplo, las herramientas móviles) de integrar la ayuda "justo-a-tiempo" y la interacción en diferentes contextos de aprendizaje. (JÄRVELÄ, 2006, p. 40).

Sin embargo, hasta el momento, el indiscutible potencial, rapidez y efectividad de esas tecnologías para recoger, gestionar, guardar y transmitir sonidos, textos, gráficoseimágenes estáticasyen movimiento, queharevolucionado muchas otras áreas (el mundo de la música, la investigación, la economía, etc.) apenas ha arañado las estructuras y las formas de hacer de la escuela.

Una de las primeras razones que se esgrimen para explicar este hecho es la dificultad que tienen los sistemas educativos, y no sólo los de los países en vías de desarrollo, de garantizar el acceso a unos recursos que son caros de adquirir y mantener y envejecen con rapidez. Ésta no es una razón baladí. Resultaría curioso ver una entidad en la que los trabajadores sólo pudieran utilizar las computadoras una o dos veces por semana y que para hacerlo tuviesen que desplazarse a un lugar especial en pequeños grupos a horas predeterminadas, no en el momento de necesitarlas. Pero esa es la situación en la que se encuentran un gran número de instituciones de enseñanza (escuelas y universidades) públicas y privadas y de países más y menos ricos. No obstante, incluso cuando las escuelas de enseñanza primaria y secundaria cuentan con un buen número de ordenadores y acceso a Internet, no todos los profesores los utilizan (BECKER, 2001; CONLON; SIMPSON, 2003; PELGRUM, 2001; PLOMB et al., 2003; SIGALÉS; MOMINÓ; MENESES, 2007; WILSO N; NOTAR; YUNKER, 2003).

En el caso de que se utilicen, los docentes encuentran dificultad en modificar sus rutinas pedagógicas y sus expectativas sobre el aprendizaje de los estudiantes. La investigación muestra de forma continua que el uso de las TIC no produce los cambios esperados en las prácticas de enseñanza y aprendizaje (CUBAN, 2001; CUBAN; KIRKPATRICK; PECK, 2001; KOZMAN, 2003; OECD , 2004; RING STAFF; KELLEY, 2002; SANCHO , et al., 2004; SCHO FIELD ; D AVID SO N, 2002).

A menudo las TIC se utilizan para reforzar la cultura y las creencias predominantes sobre la educación en las que enseñar consiste en explicar (lo haga el docente o el material multimedia), aprender consiste en escuchar (o leer la pantalla del ordenador) y el conocimiento es lo que contiene el libro (CUBAN, 1993), o ahora el software educativo.

Rev. Diálogo Educ., Curitiba, v. 9, n. 28, p. 651-669, set./ dez. 2009 
El análisis de los resultados del conjunto de investigaciones disponibles indica que el uso de las TIC en la enseñanza, cuyos desarrollos han sido generados fuera del campo de la educación con la finalidad de resolver problemas específicos del ejército (NOBLE , 1991), la economía, la investigación, o simplemente para aumentar el consumo, se enfrenta con un conjunto de factores que o no se suelen tener en cuenta o resultan tan difíciles de transformar que nadie parece interesado en poner empeño en ello. Parece que da más publicidad, y quizás rédito político, decir de que el gobierno va a gastar X miles o millones de reales en comprar "una computadora por niño o niña", que mejorar las condiciones de trabajo en las escuelas, la formación del profesorado y los programas de estudio. Pero como argumentaba el profesor Urie Bronfenbrenner, si alguien quiere entender la complejidad de un sistema, lo primero que ha de hacer es intentar cambiarlo. Y esta perspectiva es la que permite el estudio de la dificultad de las TIC para transformar (y mejorar) el complejo entramado de tecnologías artefactuales (pizarras, libros didácticos, etc.); organizativas (edificios, clases en filas y columnas, espacios, tiempos, horarios, currículos... ) y simbólicas (el lenguaje textual, modo de expresión por excelencia de la escuela,... ), que hoy constituyen la tecnología por excelencia de la educación. 0 en términos de Tyack y Tobin (1994 de la gramática de la escuela, constituida por:

Las estructuras y pautas regulares que organizan el trabajo de la enseñanza. Aquítenemospresente, porejemplo, las prácticas organizativas estandarizadas en la división del tiempo y del espacio, laclasificación del alumnado y su distribución en clases, yla fragmentación del conocimiento en 'asignaturas'. En 1902 John D ewey argumentaba que era fácil rechazar la forma en que las escuelas estaban organizadas 'como algo comparativamente externo e indiferente a las finalidades e ideales educativos', pero, de hecho 'la forma en que la maquinaria de la enseñanza influye en el niño... realmente controla todo el sistema'. La continuidad en la gramática dela enseñanza ha frustrado a generaciones de reformadores que han intentado cambiar estas formas organizativas estandarizadas. (TYACK; TOBIN, 1994, p. 454).

A partir de este posicionamiento, veamos el conjunto de factores que dificultan que las TIC se conviertan en una tecnología transformadora de la educación.

Rev. Diálogo Educ., Curitiba, v. 9, n. 28, p. 651-669, set./ dez. 2009 
La transformación de las tecnologías de la información y la comunicación en tecnologías de la educación

1. La propia naturaleza de las TIC. Las TIC (computadoras, Internet, etc.) son herramientas vacías en busca de sentido. Tienen una gran capacidad para recoger, gestionar, almacenar y transmitirgrandes volúmenes deinformación, pero necesitan de alguien que produzca esa información y sobre todo, alguien que la interprete y le dé sentido. Tener un ordenador con acceso a Internet y no saber leer o no conocer las lenguas en las que está escrita la información o los distintos lenguajes (gráfico, visual,...) que es como no tener nada. Estas tecnologías requieren (a la vez que contribuyen a crear) usuarios rápidos en entender la información y capaces de situase con criterio frente al ingente volumen de información disponible. Esto requiere tener un propósito, saber plantearse preguntas, tener elementos que permitan evaluar la fiabilidad de la información a la que se accede, saber elaborar las propias conclusiones y expresarlas mediante diferentes formatos.

Este conjunto de conocimientos, habilidades y actitudes (competencias según la nueva moda en el discurso educativo) puede desarrollarse 0 adquirirse con o sin el computador; pero su mera utilización no lo garantiza si no va acompañada de una determinada acción educativa. Y esto es así porque la naturaleza abierta de esta tecnología ha llevado a todas las tendencias educativas a ver en la computadora la gran esperanza blanca de la educación.

- las perspectivas conductistas y neoconductictas de la enseñanza han constituido a la computadora como la máquina enseñar, el sistema experto o tutor inteligente por excelencia. D esde esta visión se ha desarrollado (y se sigue desarrollando) una importante actividad de diseño y desarrollo de programas de enseñanza asistida por ordenador (software educativo);

- desde la visión de las disciplinas como articuladoras del aprendizaje, que presupone que en cada materia subyacen ciertos conceptos clave y una secuencia lógica que una vez dominadas por los estudiantes se pueden transferir o aplicar anuevas situaciones, se ve en la programación de ordenadores el mismo potencial de desarrollo del pensamiento lógico que un día detentaron el Latín y las Matemáticas. D e ahí el movimiento orientado a incluir en el currículo una asignatura de programación informática;

Rev. Diálogo Educ., Curitiba, v. 9, n. 28, p. 651-669, set./ dez. 2009 
- seymour Papert defendía el uso educativo del LO G O como la plasmación dela perspectiva constructivista del aprendizaje a partir de las ideas de Jean Piaget;

- las visiones cognitivas y neocognitivas del aprendizaje y la enseñanza ven en la computadora la metáfo ra explicativa del cerebro humano; la herramienta que transforma lo que toca y que es capaz de realizar acciones humanas (calcular, tomar ciertas decisiones, enseñar...). Para ellos, toda actividad mediada por esta máquina presupone el desarrollo de capacidades cognitivas y metacognitivas (resolución de problemas, planificación, algoritmización de tareas, etc.). De ahí la argumentación de que la experimentación y la exploración de lainformación, en cualquierárea del currículo escolar, mejorainmediatamentela motivación, el rendimiento y las capacidades cognitivas del alumnado;

- desde la concepción del aprendizaje como procesamiento de información, basado en el presupuesto de que el aprendizaje radica en la expresividad y diversificación de los códigos utilizados para representar la información en los medios de enseñanza, se ve en la capacidad de los recursos digitales para integrar textos, sonidos, gráficos y lenguaje audiovisual, la respuesta a los problemas de motivación y rendimiento del alumnado (e incluso del profesorado);

- desdela concepción del aprendizaje compartido o comunitario (HO LMES et al., 2001), basada en la convicción de que el aprendizaje se basa en el intercambio y la cooperación, la asunción de riesgos, el planteamiento de hipótesis, el contraste, la argumentación, el reconocimiento del otro y la aceptación de la diversidad, los sistemas informáticos, la navegación por la información y la ampliación de la comunicación con personas e instituciones geográficamente distantes que permiten las redes telemáticas, se ven como respuesta a las limitaciones que supone el espacio escolar.

D e ahí que la mera presencia del computador en la escuela y en el aula, no implique de forma automática no sólo un cambio en la forma de enseñar y aprender y una mejora de los resultados de aprendizaje del alumnado, sino incluso una garantía de uso cotidiano de la herramienta. Porque además la práctica docente no se realiza en el vacío sino que está sumergida en la organización y la cultura inercial de la escuela.

Rev. Diálogo Educ., Curitiba, v. 9, n. 28, p. 651-669, set./ dez. 2009 
La transformación de las tecnologías de la información y la comunicación en tecnologías de la educación

2. La cultura de la escuela. Como hemos señalado anteriormente de la mano de Tyack y Tobin (1994), la historia de la innovación educativa nos ha enseñando lo difícil que es cambiar las normas de la gramática de la escuela. De hecho, uno de los resultados de un proyecto europeo realizado con la finalidad de incidir en la mejora de la escuela desde una visión pedagógicamente integrada del uso de las TIC, fue poder identificar un conjunto de factores institucionales que dificultan el uso de estas herramientas. Entre ellos encontramos:

a) las especificaciones disciplinares y la forma en la que están establecidos los niveles de logro de los currículos actuales;

b) las restricciones que provienen de la propiaAdministración, en términos de dotación presupuestaria, autonomía de las escuelas, etc;

c) los esquemas organizativos imperantes de la enseñanza (lecciones de 45-50 minutos);

d) la organización del espacio - acceso a las computadoras, número de estudiantes por clase, etc.;

e) los sistemas de formación permanente del profesorado, demasiado centrados en la herramienta, que impiden el cambio educativo;

f) el contenido disciplinar de los currículos que dificulta las propuestas transdisciplinares y el aprendizaje basado en problemas;

g) la falta de motivación por parte del profesorado para introducir nuevos métodos;

h) la poca autonomía que gozan el profesorado y el alumnado (SANCHO, 2006).

D ehecho, muchos delos elementos que configuran latecnología de la educación de las escuelas están configurados por las políticas educativas vigentes.

3. La política educativa. Si existe un ámbito de actuación dependiente de las políticas gubernamentales éste es el de la educación escolar. Basta con observar, en la mayoría de los países, cómo cada nuevo gobierno intenta una reforma de la escuela. 0 cómo, en los últimos años, los responsables

Rev. Diálogo Educ., Curitiba, v. 9, n. 28, p. 651-669, set./ dez. 2009 
políticos tienden a invertir en TIC, algo que les da presencia mediática, sin tener en cuenta los problemas reales de las escuelas reales, el profesorado real, y los estudiantes y las familias reales. D e ahí que en estemomento estemos realizando un proyecto titulado Políticas y prácticas en torno a las TIC en la enseñanza obligatoria: Implicaciones para la innovación y la mejora. ${ }^{8}$

Uno de los objetivos del proyecto es describir, analizar e interpretar el sentido educativo de las políticas institucionales puestas en práctica en Cataluña (España) destinadas a facilitar la incorporación y uso de las TIC al sistema escolar en los últimos 20 años.

El análisis crítico del discurso fijado en los documentos institucionales que configuran la política desarrollada en estos años y en las 12 entrevistas que hemos realizado a otros tantos responsables de la Administración, nos ha permitido llegar a las siguientes conclusiones:

a) la política orientada a la integración de las TIC en la educación desconsidera el importante volumen de conocimiento disponible sobre el cambio educativo y carece de una teoría o perspectiva explícita o implícita de cambio educativo;

b) las TIC son consideradas como herramientas potentes, cuya utilización será capaz de transformar la enseñanza, a pesar de que todo el resto del sistema permanezca igual;

c) la política específica para fomentar el uso educativo de las TIC no establece ninguna conexión con otras iniciativas para promover la innovación y la mejora de la escuela;

d) la políticallevada a cabo hasta el momento tiene poco o nada en cuenta los resultados de las investigaciones realizadas sobre el uso de las TIC en la enseñanza;

e) lasinversiones económicas se realizan de formafragmentaria y el equipamiento de las escuelas no suele estar relacionado con proyectos de innovación docente;

f) no se presta atención a las iniciativas generadas desde el propio profesorado;

g) por depender de entidades gubernamentales diferentes, no se considera la formación inicial del profesorado;

8 Políticas y prácticas en torno a las TIC en la enseñanza obligatoria: Implicaciones para la innovación y la mejora. Ministerio de Ecuación y Ciencia. SEJ2007-67562. Accesible at: <http:/ / fint.doe.d5.ub.es/ fint2005/ index.php?page=tic>.

Rev. Diálogo Educ., Curitiba, v. 9, n. 28, p. 651-669, set./ dez. 2009 
La transformación de las tecnologías de la información y la comunicación en tecnologías de la educación

En cuanto la formación permanente, que sí es responsabilidad de esta iniciativa política:

a) las actividades de formación están claramente orientadas al conocimiento de la herramienta y sus aplicaciones (software educativo, lenguajes de programación, páginas web, Web2.0, etc.) dejando totalmente de lado los elementos de integración curricular;

b) son de carácter genérico, por lo que no tienen en cuenta las necesites educativas de los distintos estudiantes;

c) no promueven la comprensión crítica de los fenómenos de la Sociedad de la Información y los desafíos que plantean a la educación;

d) no facilitan que el profesorado adquiera y desarrolle un marco conceptual para entender los cambios de rol de docentes y alumnos en un mundo saturado de información;

e) se dirigen fundamentalmente a profesores individuales, no a las escuelas como sistemas sociales complejos.

En definitiva, para que las TIC lleguen a convertirse un día en una TecnologíaE ducativa que realmente transformey mejorela ecuación, habría que ampliar la mirada sobre lo que significa hoy aprender y enseñar en un mundo al que le sobra información y le falta sentido.

\section{CONCLUSIONES}

En los últimos años, el uso de las computadoras y de los distintos desarrollos vinculados a las tecnologías digitales de la información y la comunicación han sido presentados no sólo como las herramientas por excelencia para la mejora de la educación, sino como factor de modernización de los sistemas educativos. Sin embargo, el seguimiento de los distintos proyectos llevados a cabo y las inversiones realizadas, a menudo a costa de deuda externa, no han podido evidenciar que su uso en la escuela signifique una mejora inmediata de los procesos de enseñanza y aprendizaje y de los resultados de los estudiantes.

Un análisis sistémico de la complejidad de los sistemas escolares revela que centrar la mirada en los recursos tecnológicos sin tener en cuenta el resto de los componentes que configuran el sistema, impide utiliz ar el potencial de estas tecnologías en experiencias de aprendizaje que

Rev. Diálogo Educ., Curitiba, v. 9, n. 28, p. 651-669, set./ dez. 2009 
realmente valgan la pena para el profesorado y el alumnado. El resultado de esta tendencia es que las inversiones, a veces millonarias, tienen el mismo efecto que el que produce echar agua en la arena de la playa. La consecuencia de este efecto no es sólo que las escuelas no mejoren, sino que cada vez sean más pobres (si consideramos la falta de inversión en otros recursos - humanos y materiales- esenciales) y tengan más chatarra (dada la velocidad con la que envejecen los aparatos).

Sólo una visión integral del conjunto de factores que configuran la educación escolar y una actuación conjunta de los diferentes agentes que intervienen en él podrá facilitar el camino de las TIC hacia el desarrollo de una Tecnología de la E ducación, capaz de responder a los múltiples desafíos a los que se enfrenta la educación actual.

\section{REFERÊNCIAS}

BALANSKAT A.; BLAMIRE R.; KEFALA, S. The ICT Impact Report. A review of studies of ICT impact on schools in Europe. 2006. Accesible at: <http:/ / insight.eun.org/ shared/ data/ pdf/ impact_study.pdf>.Consultado el: 21 mayo 2007.

BECKER, H. How are teachers using computers in instruction? University of California, Irvine, 2001. Paper presented at the 2001 Meetings of the American Educational Research Association. Accesible at: < https:/ / www.msu.edu/ course/ cep/ 807/ zO ld807.1998G entry/ snapshot.afs/*cep240studyrefs/ beckeraera2001howtchrsusing.pdf>. Consultado el: 23 ago. 2009.

BIGUM, C.; KENWAY,J. New information technologies and the ambiguous future of schooling - some possible scenarios. In: HARGREAVES, A. etal. (Ed.). Intemational handbook of educational change. D ordrecht: Sptimger, 1998. p. 95-115.

CARSTENS, A.; BECK, J. Get ready for the gamer generation. TechTrends, Washington, D. C., v. 49, n. 3, p. 22-25, 2005.

CONLON, T.; SIMPSO N, M. Silicon valley verses silicon glen: the impact of computers upon teaching and learning: a comparative study. British Journal of Educational Technology, London, v. 34, n. 2, p. 137-150, 2003.

CUBAN, L. How teachers taught: constancy and change in American classrooms, 1890-1990. New York: Teachers College Press, 1993.

Oversold and underused: computers in the classroom. Cambridge, Mass.: Harvard University Press, 2001.

Rev. Diálogo Educ., Curitiba, v. 9, n. 28, p. 651-669, set./ dez. 2009 
La transformación de las tecnologías de la información y la comunicación en tecnologías de la educación

CUBAN, L.; KIRK PATRICK, H.; PECK, C. High access and low use of technologies in high school classrooms: Explaining an apparent paradox. American Educational Research Journal, Washington, D. C., v. 38, n. 4, p. 813-834, 2001.

HOLMES, B. et al. Communal constructivism: students constructing learning for as well as with others. In: INTERNATIONAL CONFERENCE OF THE SOCIETY FOR INFORMATION TECHNOLOGY \& TEACHER EDUCATION (SITE), 12th, 2001, Charlottesville, VA. Proceedings... Charlottesville, VA: Association for the Advancement of Computing in Education, 2001.

ISTANCE, D. Los escenarios de la escuela de la OCDE, el profesorado y el papel de las tecnologías de la información y la comunicación. In: SANCHO, J. M. (Coord.). Tecnologías para transformar la educación. Madrid: AKAL/ UNIA, 2006. p. 233-261.

JÄRVELÄ, S. Personalised learning? New insights into fostering learning capacity. In: _. (Ed.). Personalising education. Paris: OECD/ CERI, 2006. p. 31-46.

KOZMAN, R. B. Technology, innovation, and educational change - a global perspective. Washington, D. C.: ISTE, 2003.

LENHART, A.; RAINIE , L.; LEWIS, O. Teenage life online: the rise of instantmessage generation and the internet's impact on friendship and family relationships. Washington, D.C.: Pew Internet \& American Life Project, 2001.

McKeNZIE, J. Putting an end to topical research. From Now 0n. The Educational Technology Journal, v. 16, n. 3, Feb. 2007. Accesible at: <http:/ / www.fno.org/ feb07/ topic.html>. Consultado en: 23 ago. 2009.

NOBLE, D. D. The classroom arsenal: military research, information technology and public education. London: The Falmer Press, 1991.

OBLINGER, D.; OBLINGER, J. L. (Ed.). Educating the Net Generation. Washington, D.C.: Educause, 2005.

ORGANISATION FOR ECONOMIC CO-OPERATION AND DEVELO PMENT - OECD. Education at a Glance 2004. Paris: OECD, 2004.

OPPENHEIMER, T. The flickening mind. saving education from the false promise of technology. New York: Random House, 2004.

PAPERT, S. Computers and learning. In: DERTOUZO S, M. L.; MO SES, J. (Ed.). The computer age: a twenty-year view. Cambridge, Mass.: MIT, 1979. p. 80-96.

Rev. Diálogo Educ., Curitiba, v. 9, n. 28, p. 651-669, set./ dez. 2009 
PELGRUMN, W. J. Obstacles to the integration of ICT in education: results from a worldwide educational assessment. Computers \& Education, New York, v. 37, p. 163-187, 2001.

PERELMAN, L. J. School's out, hyperlearning, the new technology, and the end of education. New York: William Morrow and Company, Inc., 1992.

PLO MP, T. et al. (Ed.). Cross-national policies and practices on information and communication technology in education. $G$ reenwich, $\mathrm{CT}$ : Information Age Publishing Inc., 2003.

RING STAFF, C.; KELLEY, L. The learning return on our educational technology investment. A review of findings from research. WestEd, San Francisco, p. 1-30, 2002. Accesibleat: <http:/ / www.wested.org/ cs/ we/ view/ rs/ 619>. Consultado el:2 nov. 2003.

SANCHO, J. M. Enfoques y funciones de las nuevas tecnologías para la información y la educación: lo que es no es lo que parece. In: PABLOS, J. de.; JIMÉNEZ, J. (Coord.). Nuevas tecnologías. Comunicación audiovisual y educación. Barcelona: Cedecs, 1998. p. 71-102.

De tecnologias da informação e a comunicação a recursos educativos. In: SANCHO, J. M.; HERNÁNDEZ, F. (Coord.). Tecnologias para transformar a educação. Porto Alegre: Artmed, 2006. p. 15-41.

Digital technologies and educational change. In: HARG REAVES, A. et al. (Ed.). International handbook of educational change. D ordrecht: Boston; London: Springer. En prensa.

SANCHO, J. M.; HERNÁNDEZ, F. (Ed.). Tecnologias para transformar a educação. Porto Alegre: Artmed, 2006.

SANCHO, J. M. et al. Final report. School + more than a platform to build the school of tomorrow. Luxembourg: European Commission, 2004.

SCHO FIELD, J. W.; DAVID SO N, A. L. Bringing the internet to school: lessons from an urban district. San Francisco: Jossey-Bass, 2002.

SIGALÉS, C.; MOMINÓ , J. M.; MENESES, J. L'escola a la societat de la X arxa. Internet a l'educació primària i secundaria. Informe final de recerca. Barcelona: Universitat O berta de Catalunya; Internet Interdisciplinary Institute (IN3), 2007. v. 1. A ccesible at: <http:// www.uoc.edu/ in3/ pic/ cat/ escola_xarxa/ informe.html>. Consultado en: 23 ago. 2009.

Rev. Diálogo Educ., Curitiba, v. 9, n. 28, p. 651-669, set./ dez. 2009 
La transformación de las tecnologías de la información y la comunicación en tecnologías de la educación

TYACK, D.; TO BIN, W. The "G rammar" of schooling: Why has it been so hard to change? American Educational Research Journal, Washington, D. C., v. 31, n. 3, p. 453-480, 1994.

VEEN, W.; VRAKKING, B. Homo Zappiens: growing up in a digital age. London: Continuum International Publishing G roup, 2006.

WILSO N, J. D.; NOTAR, C. C.; YUNKER, B. Elementary in-service teacher's use of computers in the elementary classroom. Journal of Instructional Psychology, D ec. 2003. Accesible at: <http:/ / www.findarticles.com/ p/ articles/ mi_m0FCG/is_4_30/ai_112686159>. Consultado en: 23 ago. 2009.

Recebido: 10/ 02/ 2009

Received: 02/ 10/ 2009

Aprovado: $15 / 03 / 2009$

A pproved: 03/ 15/2009

Revisado: 15/ 09/ 2009

Reviewed: 09/ 15/ 2009

Rev. Diálogo Educ., Curitiba, v. 9, n. 28, p. 651-669, set./ dez. 2009 